\title{
Microscopic diamond Solid-Immersion-Lenses fabricated around single defect ceneters by focussed ion beam milling
}

\author{
Mohammad Jamali, ${ }^{1}$ Ilja Gerhardt, ${ }^{1,2}$ Mohammad Rezai, ${ }^{1}$ Karsten Frenner, ${ }^{3}$ Helmut \\ Fedder, ${ }^{1}$ and Jörg Wrachtrup ${ }^{1,2}$ \\ 1) 3. Physikalisches Institut and Stuttgart Research Center of Photonic Engineering \\ (SCoPE), Universität Stuttgart, Pfaffenwaldring 57, Stuttgart D-70569, \\ Germany \\ 2) Max Planck Institute for Solid State Research, Heisenbergstraße 1, \\ D-70569 Stuttgart, Germany \\ ${ }^{3)}$ Institute for technical optics (ITO), Universität Stuttgart, Pfaffenwaldring 7 , \\ Stuttgart D-70569, Germany
}

(Dated: 13 October 2014)

Recent efforts to define microscopic solid-immersion-lenses (SIL) by focused ion beam milling into diamond substrates that are registered to a preselected single photon emitter are summarized. We show how we determine the position of a single emitter with at least $100 \mathrm{~nm}$ lateral and $500 \mathrm{~nm}$ axial accuracy, and how the milling procedure is optimized. The characteristics of a single emitter, a Nitrogen Vacancy (NV) center in diamond, are measured before and after producing the SIL and compared with each other. A count rate of 1.0 million counts per second is achieved with a [111] oriented NV center. 


\section{INTRODUCTION}

Single optical emitters embedded in solid state materials are on the research horizon for more than twenty years ${ }^{1}$. Not only their single photon emission, but also their nanoscopic size, and their properties as single optical and magnetic qubits allow for various quantum optics, quantum information and sensing experiments. Among them, color centers in diamond especially the negatively charged nitrogen vacancy (NV) center emerges as one of the promising candidates. It has a strong optical transition at $637 \mathrm{~nm}$ and local electron and nuclear spins with long spin coherence times even at room temperature that are suitable for quantum memories. Therefore, it has been used in different applications, such as quantum registers, magnetic field sensors and diamond-based single photon sources ${ }^{2-6}$.

Generally, a solid state environment allows for high collection efficiencies ${ }^{7,8}$. The acceptance angle into the collection optics can be very high, and high refractive index allows for a small focus size. Various methods to increase collection efficiency have been proposed and experimentally studied ${ }^{9}$. Among them, thin layers ${ }^{8}$, pillar structures ${ }^{10}$, and solid immersion lenses (SILs) have been explored ${ }^{11}$. A solid immersion lens increases the collection efficiency from a single emitter, by circumventing refraction from interfaces and thereby increasing the numerical aperture. Different geometries, namely the hemispherical SIL and the Weierstrass SIL have been researched ${ }^{9}$. The later is not optimal for spectrally broad emitters, since the shape results in strong chromatic abberations. Hemispherical solid immersion lenses have been successfully used in single emitter studies, e.g. with single molecules ${ }^{12}$, quantum $\operatorname{dots}^{13}$ and single defects in solids ${ }^{14-16}$.

Using a SIL for luminescent defects in diamond is especially interesting, since diamond has one of the highest refractive indices in the visible range $\left(n_{d}=2.42\right)$. The refractive index difference at the diamond-air interface causes strong refraction and total internal reflection (critical angle $24^{\circ}$ ) for the emitted light. Therefore, light emitted by a defect cannot be efficiently collected. Also for ideal spin properties, the NV defects under study should be embedded deeply in substrate ${ }^{17}$. Hence, it is important to fabricate special optical structures to enhance collection efficiency.

The approach of producing SILs directly on diamond started recently ${ }^{18}$. Two approaches have been established: To produce macroscopic half-spheres, with length scales of milimeters $^{18}$, and to produce microscopic SILs in the order of serval micrometers ${ }^{14,19}$. Macroscopic 
SILs were produced by laser and mechanical processing from small single crystalline CVD diamonds, which are overgrown on high quality high temperature high pressure (HPHT) grown diamond substrate. Microscopic SILs can be produced by focused ion beam (FIB) milling.

This paper outlines the microscopic manufacturing process for SILs. We describe the pathway to manufacture a solid immersion lens: first, a single emitter is optically located and characterized. Afterwards a SIL is manufactured around it. This is achieved by FIB milling. We compare different milling strategies and present the one that is optimal to produce SILs closest to the desired hemispherical shape and with least milling residuals.

\section{LOCATING A SINGLE EMITTER}

Before producing a SIL around a single emitter, we have to locate the emitter in all three spatial directions. Since these steps, the characterization under confocal microscopy and milling in the FIB machine, are performed in two different setups, it is required to introduce suitable marker structures that are visible in both microscopes and to which the position of the emitter and the SIL are referenced. This is required for lateral localization. For the localization in depth, we do not require a retrievable structure, since the surface serves as a reference.

We first discuss the lateral localization of the emitter: The localization accuracy of a single NV should be in the range of the field of view of the SIL. The field of view diameter, $d_{F O V}$, follows from a quater-wave criterion ${ }^{20}$ and is proportional to the square root of the SIL radius, $r$.

$$
d_{F O V}<\sqrt{\frac{2 r \lambda}{n(n-1)}},
$$

where $\lambda$ is the optical wavelength and $n$ is the refractive index of the SIL. For a $4 \mu \mathrm{m}$ SIL in diamond and for $\lambda=532 \mathrm{~nm}$ this is about $1 \mu \mathrm{m}$. Consequently, the ability to locate an emitter, has to be significantly better then $1 \mu \mathrm{m}$, both in the FIB machine and the optical microscope.

We locate the emitter by measuring its relative position against three marker points in the confocal microscope, further we locate these points under the FIB and calculate the actual position of the NV. For this purpose we mill a rectangular pattern of cylindrical holes 
(diameter $260 \pm 20 \mathrm{~nm}$, depth $500 \mathrm{~nm}$, pitch $20 \mu \mathrm{m}$, current $0.92 \mathrm{nA}$ ) into the sample with the FIB. These markers are well visible in the confocal fluorescence microscope (Fig.1a,b). It is currently not clear what is at the origin of their fluorescence. The fluorescence could either stem from graphitized material, implanted gallium or dirt contained in the immersion oil that is trapped inside the holes. Single emitters are laterally located by imaging the sample at the target emitter depth below the surface with a home-made confocal microscope (Fig.1c), including an oil-objective (Olympus, UPLANSAPO, 60×, $1.35 \mathrm{NA}$ ), single photon counting detectors and a $585 \mathrm{~nm}$ long-pass filter. The sample is mounted on a piezo scanner (PI, P-517.3CD with $1 \mathrm{~nm}$ in-plane and $0.1 \mathrm{~nm}$ vertical resolution). The excitation power was $0.5 \mathrm{~mW}$ onto the diffraction limited spot ( $600 \mathrm{~nm}$ ). The imaging depth corresponds to the desired SIL radius, typically $2-6 \mu \mathrm{m}$ in the present case. Single emitters were identified by measuring the autocorrelation function in a Hanbury-Brown and Twiss configuration. After the emitters were located, the surface of the sample was imaged to locate the position of the three holes around the NV accurately. The accuracy of fabricated SIL is limited by the accuracy of locating markers in both experimental configurations. Since the optical signal from a single marker originates from a sub-wavelength structure, the accuracy for locating an ideal point source $\sigma_{r}$ is in theory on the order of $\sigma_{r} \sim 0.61 \lambda /(N \cdot A \cdot \sqrt{N})$, where $N$ is the number of detected photons. This amounts to $\sim 1 \mathrm{~nm} / \sqrt{\mathrm{Hz}}$ for a single emitter with a detected count rate of 100 kcounts/s. For non-ideal spherical sources, such as the present alignment markers or for non-axial dipoles such as the single emitters in the present case, the localization accuracy is lower. The alignment accuracy is further limited by a (possibly inhomogeneous) fluorescence background and drift as well as imperfect repeatability of the piezo scanner. To examine the accuracy of this alignment procedure, we made the following test. First, we identified a specific emitter, then three markers around it were selected and all coordinates were recorded. As next step, the procedure was repeated and the coordinates of the same markers and emitter were recorded again. Finally, the expected coordinates of the emitter were calculated from the previous coodinates of the emitter and the previous and new coordinates of the markers. This procedure is identical to the calculation of the target SIL position from the ion beam image. The difference between the calculated and the measured new position of the emitter defines roughly the positioning accuracy which was typically better than $100 \mathrm{~nm}$. In fact, by minimizing mechanical drift and fitting the experimental data with a Gaussian function it should be possible to achieve positioning accuracy of several 


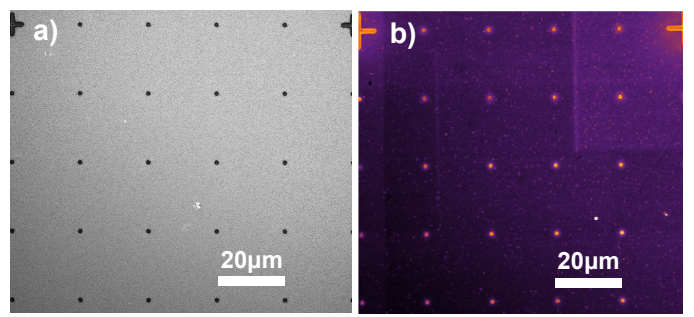

c)

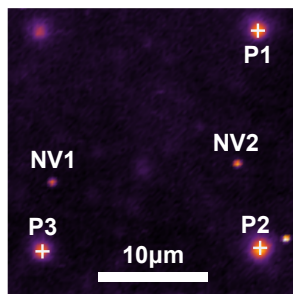

FIG. 1. a) SEM image of focused ion beam generated markers in a rectangular pattern of $20 \times 20 \mu \mathrm{m}$. Pixel size: $120 \mathrm{~nm}$. b) Optical image of the same markers, acquired in a confocal microscope. Pixel size: $200 \mathrm{~nm}$. c) Confocal image of Sample showing two NVs and the markers around them. Note that the markers appear larger since the focal plane coincides with the NVs such that the markers are out of focus.

nanometers ${ }^{21,22}$. As there was no need for such a accuracy in our experiment, the positions of the markers were extracted directly from the brightest pixels in the image.

The lateral coordinates $r_{e}^{\prime}$ of the emitter in the ion image follow from the coordinates of the emitter $r_{e}$ and the coordinates of the markers $r_{0}, r_{1}, r_{2}$ in the optical image and the coordinates of the same markers $r_{0}^{\prime}, r_{1}^{\prime}, r_{2}^{\prime}$ in the ion image as

$$
r_{e}^{\prime}=r_{0}^{\prime}+V^{\prime} V^{-1}\left(r_{e}-r_{0}\right)
$$

where the rows of the $2 \times 2$ matrices $V$ and $V^{\prime}$ are $r_{1}-r_{0}$ and $r_{2}-r_{0}$, respectively $r_{1}^{\prime}-r_{0}^{\prime}$ and $r_{2}^{\prime}-r_{0}^{\prime}$.

After discussing the lateral localization of the emitter, we now turn to the depth determination. Optically, this is not a trivial task, since the refractive index mismatch between the immersion oil and the diamond elongates the effective focal length of the microscope objective and distorts its point spread function. Consider the extremal rays, which are given by the numerical aperture of the objective lens as shown in Fig. 2a). The depth of the emitter $d$ follows from the displacement $d_{0}$ of the piezo stage as $d=d_{0} \tan \theta_{0} / \tan \theta$, where $\theta=\arcsin \left(\frac{n_{0}}{n} \sin \theta_{0}\right)$ and $n_{0} \sin \theta_{0}=N . A$., where $n_{0}$ is the refractive index of the surrounding medium and $\theta_{0}$ is the half opening angle. It is obvious that every ray bundle in the light 
cone results in a different focal shift. This is a well known problem in confocal microscopy studied both experimentally ${ }^{23,24}$ and theoretically ${ }^{25}$. For low numerical aperture lenses and small refractive index mismatch the estimate based on the extremal rays is reportedly a good approximation ${ }^{23,25}$. With high numerical aperture lenses this approximation should be replaced by a wave optical treatment and the distorted point spread function should be computed. The intensity maximum of the point spread function (PSF) would then be identified with the apparent focus position. Note that in the present case of confocal fluorescence microscopy of a single emitter a number of additional effects should be taken into account, including both the illuminating and the emitting field $(532 \mathrm{~nm}$ and 650-750 nm, respectively, in the present case), which are additionally effected by chromatic aberations, the pinhole, and the emission pattern, determined by two perpendicular dipoles in case of the NV center. Moreover, in the present case the refractive index mismatch between the immersion oil $\left(n_{\text {oil }}=1.52\right)$ and diamond is exceptionally large. Note that in a full wave model, the effective depth will also no longer depend linearly on the nominal depth. Generally, as the PSF gets more and more distorted and the imaging quality is reduced, the objective lens effectively behaves like a lens with smaller numerical aperture. Or, equivalently, the extremal rays will no longer result in constructive interference and contribute less. We now perform two rough estimates to account for these effects: on the one hand, we consider the effective depth corresponding to the mean opening angle, given by $\bar{\theta}=0.5 \arcsin (N . A . / n)$. On the other hand, we evaluate the effective depth averaged over all rays that impinge on the back aperture of the objective with equal weight. The latter corresponds to a simple ray traycing model. In the present case, we obtain a correction factor $d^{\prime} / d=\gamma=1.80$ and $\gamma=2.42$, for the prior and the latter estimate, respectively. We have also checked these results by full wave optical simulations of the three-dimensional PSF using Zemax, assuming an ideal objective lens with the same N.A. as the one used in experiments. In this approach we used the same wavelength $(650 \mathrm{~nm})$ for illumination and emission and disregarded the confocal pinhole. These simulations confirmed that the conversion factor is depth dependent and in the range $\gamma \in[2.10,1.95]$ for $d_{0} \in[0,10] \mu \mathrm{m}$. For the experiments $\gamma=1.85$ was used, which led to good results. While not being subject of the present study one could extend on this. The effective focus depth could be determined experimentally as follows. Consider a diamond sample with a thin (few $\mathrm{nm}$ ) fluorescent layer at a depth several micrometers below the sample surface (such a sample could be created e.g. by CVD growth and delta doping ${ }^{26}$ 
a)

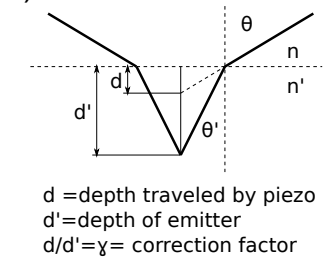

b)

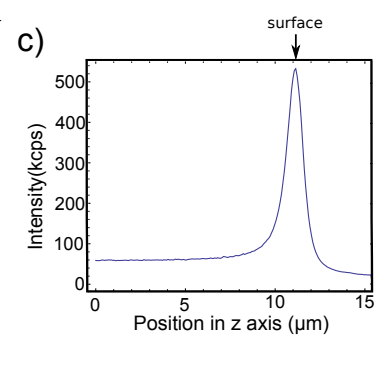

FIG. 2. a) Schematic of the focal shift in a high refractive index sample. b) Confocal scan of sample in xz plane. c) Intensity profile in z direction.

or by overgrowing a substrate with a 'dirty' initial surface). Next a staircase structure with well calibrated step height could be milled into the diamond down to the fluorescent layer by FIB. By measuring the effective depth of the fluorescent layer on all steps, the depth dependent effective focus could be reconstructed.

The other crucial factor for the depth detecrmination is to locate the sample surface precisely. For this purpose we obtained a x-z scan through the sample (Fig.2b). The confocal image shows a bright band with a narrow line of higher intensity at its center, also the plot of the $\mathrm{z}$ position versus the intensity of the light shows a sharp peak which is related to the surface (Fig.2c). Our experimental results prove that our accuracy in depth determination is better than $1 \mu \mathrm{m}$.

We produce hemispherical SILs with the radius equal to the depth of the NV, and a cone surrounding the SIL (Fig.4). The cone is chosen slightly larger than the acceptance cone of the microscope objective, such that the largest possible amount of light can be captured. More specifically, we use a cone radius slightly larger than

$$
\frac{R_{\text {cone }}}{R_{\text {SIL }}}=\tan \left(\arcsin \left(\frac{N \cdot A .}{n^{\prime}}\right)\right)
$$

where $n^{\prime}$ is the refractive index of the diamond. This ratio is equal to 2.1 for the oil objective with the N.A. of 1.35 . 


\section{FIB MILLING}

Prior to milling the sample was mounted on a conductive holder with conducting silver paste and covered with $20 \mathrm{~nm}$ conductive gold. Subsequently the sample is placed inside the FIB machine and aligned by electron microscopy (EM). Before milling, astigmatism of the ion beam was carefully aligned in a prior step to optimize the beam shape.

FIB milling was performed on an FEI, Helios 400 machine using a so-called streamfile input. This file-format is machine-specific and contains milling times and $x$ and $y$ coordinates. We wrote scripts in the python programming language to generate stream file from NV and markers positions, that we include in the supplementary material. The programs use the width of the FIB image as a reference to calculate the relative coordinates of the structure. The milling time for each point is computed based on the given milling rate and the beam current that is used for the milling.

To define a hemisphere along with a conical cutout, we milled concentric rings with decreasing inner and outer diameter. Within each ring, we stear the beam on a double spiral beam path (two interleaved spirals with opposite handedness; see also supplementary source code) with equidistant points and we adjust the milling times of the edge points to account for the spherical shape. Note that a single spiral should yield comparable results. The number of rings (layers) varies depending on the beam current and size of the SIL. We ensure that the thickness of each milling layer is much smaller than the optical wavelength. To ensure homogeneous milling and at the same time keep the memory usage in the machine within the limits, each layer may comprise several repetitions of the same path. We also tested milling with automated drift correction, however, this did not effect on the quality of the SILs.

With these settings, first the alignment holes are milled as described above. After this milling, the sample is sonicated in acetone for $10 \mathrm{~min}$ to remove silver paste that was used for mounting the sample. Then the sample is cleaned in aqua regis to dissolve the evaporated gold layer. Furthermore the sample was cleaned in piranha solution, mixture of 1:1 concentrated sulfuric acid and $30 \%$ hydrogen peroxide solution, to remove the organic material from surface and finally, rinsed in deionized water.

There are generally two milling strategies to define a 3D structure as we illustrate schematically in Fig. 3). In a first strategy, $N$ identical milling layers are used. Each milling layer 


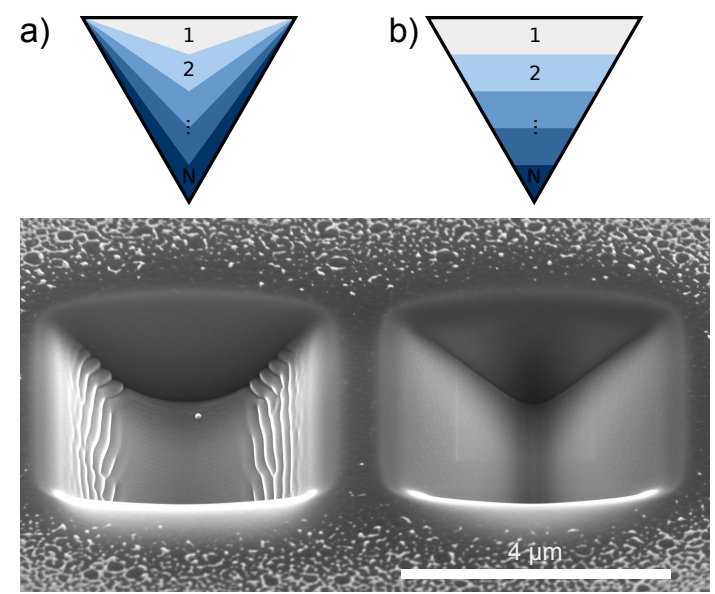

FIG. 3. Milling strategy for better focus and less residual. a) Same path, as shown schematically, was repetitively applied to the sample to mill a structure. The tip of the structure is round and there are visible residuals in both side walls. b) Different approach to mill the same structure: as shown schematically different milling paths were applied layer by layer. The sharpness is increased and no residuals are visible on the substrate.

covers the entire area of the structure and for each milling layer, the depth at each $x-y$ coordinate is $z(x, y) / N$. In a second strategy, the structure is sliced into $N$ layers of equal thickness $N / z_{\max }$ (where $z_{\max }$ is the deepest point of the structure). The layers cover different areas and are milled successively, starting with the layer that covers the largest area. In the present case, it was crucial to use the latter strategy. The effect can be seen with a $\mathrm{V}$-groove as a test structure. As is shown in Fig. 3, the first milling strategy results in significant broadening and milling residuals. By contrast, the second milling strategy produces a better result. In this case, the rounding of the dip is roughy given by the beam waist. For all milled SILs the second milling strategy was used.

The markers, which were previously located with confocal microscopy, were imaged by EM and FIB with lateral resolution of about $30 \mathrm{~nm}$. After the determination of the marker coordinates in the FIB image, the relative position of the target NV is calculated in the FIB coordinates and a stream file for a SIL with radius coinciding with the NV depth is executed using the calculated lateral NV coordinate as origin. A typical set of milling parameters for a $10 \mu \mathrm{m}$ SIL is outlined in Table I. One of the crucial values is the milling rate. To calibrate this rate we drilled a cylindrical structure on the sample with the known diameter, milling time and beam current. After milling, the depth of the cylinder was measured under $52^{\circ}$ 
TABLE I. FIB parameters

\begin{tabular}{|l|l|}
\hline Parameter & value for $10 \mu \mathrm{m}$ SIL \\
\hline \hline width of FIB image & $64 \mu \mathrm{m}$ \\
\hline milling rate & $0.21 \frac{\mu \mathrm{m}^{3}}{\mu s \cdot n A}$ \\
\hline beam current & $2.7 \mathrm{nA}$ \\
\hline radius (SIL) & $5 \mu \mathrm{m}$ \\
\hline radius (cone) & $2.2 \cdot$ radius (SIL) \\
\hline number of slices & 100 \\
\hline
\end{tabular}

angle by the SEM to calculate the volume of milled area. Some of the generated stream files have a length of 12.8 million points to be cut. The FIB only allows for a maximum number of 8 million points per file. To overcome this limitation, stream files were automatically split into several files. The produced files can be loaded into the machine at the same time and ran automatically after each other. The total milling time for the given example is 62 minutes and 55 seconds. The SEM image of the milled SIL is shown in Fig. 4a). After the milling process, the SIL is characterized in the EM. The presented SIL is a typical example and shows the quality and the overall deviations of the structure from the ideal shape. The surface roughness is determined to be on the order of $30 \mathrm{~nm}$ peak-to-valley, which was determined by SEM. In early experiments we milled cross sections through the SIL and carefully checked the spherical shape. The present stream files do not require any further corrections regarding the spherical shape of the SILs. Before any further optical characterization, the cleaning processes as explained before was applied. Additionally, the sample was boiled 3 hours in the mixture of concentrated sulphuric acid, nitric acid and perchloric acid in a volume ratio 1:1:1 to remove the residual materials and implanted gallium. While this cleaning procedure removes all background fluorescence, likely there is still implanted gallium present within the first 10 nanometers underneath the diamond surface ${ }^{27}$.

\section{OPTICAL CHARACTERIZATION}

The resulting SILs were characterized optically in a confocal fluorescence microscope. For our studies, we used a [111] cut diamond and a single NV center oriented perpendicular 


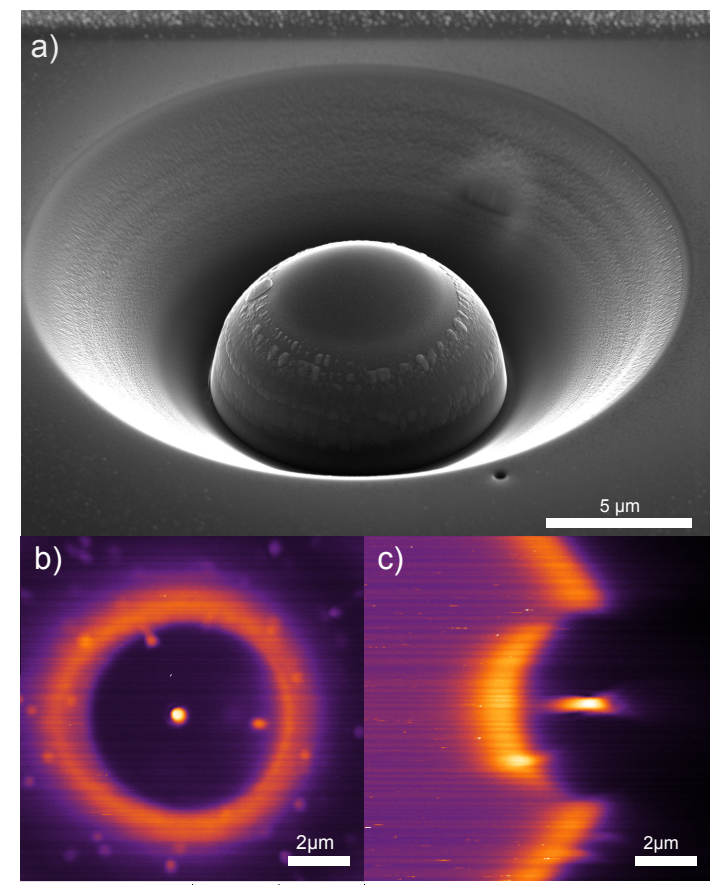

FIG. 4. SEM and optical characterization of the milled SIL. a) SEM image under $52^{\circ}$ angle. b,c) Confocal images of the SIL with the NV center in the lateral and cross sectional plane respectively.

to the diamond surface. In this case, the two optical dipoles are oriented parallel to the surface, resulting in an optimal directivity of the dipolar emission pattern. Since we have studied all emitters prior to FIB-milling, it is possible to compare the results prior and after the milling process. The central aim is to optimize the detectable count-rates. Optical studies after milling are presented in Fig. 4b) and c). The deviation of the emitter from the center of the SIL is less than $150 \mathrm{~nm}$. The PSF is found to be circular. In the linear excitation regime, the signal to background ratio is larger than 30. At higher laser powers, the background dominates, since it rises linearly with excitation power, while the single emitter is saturated. A saturated count rate of $1.0 \mathrm{Mcounts} / \mathrm{s}$ was observed, utilizing an oil objective (Fig. 5a), which is the highest count rate reported for a single NV in a bulk diamond so far. Compared to the saturated count rate without the SIL, 350 kcounts/s (data not shown), this is an enhancement $\xi=3.4$. This agrees well with the expected enhancement

$$
\xi=\frac{1-\cos (\arcsin (N \cdot A \cdot / n))}{1-\cos \left(\arcsin \left(N \cdot A \cdot / n^{\prime}\right)\right)}=3.3 .
$$

Here $n$ and $n^{\prime}$ are the refractive indices of the oil and diamond, respectively. The optical characterization was repeated with an air objective (Nikon, CFI LU Plan Fluor EPI P 100×, $0.9 \mathrm{NA}$ ), were we observed count rates of $65 \mathrm{kcnts} / \mathrm{s}$ and $600 \mathrm{kcnts} / \mathrm{s}$, without and with 
the SIL, respectively. Based on the specified numerical apertures, the collection angle of the oil and air objectives should be the same. This substantial difference in count rate cannot be explained by the increase of the reflection losses at the air-diamond interface, which is less than $10 \%$. A possible explanation for this observation could be slight deviations of the SIL from the ideal hemispherical shape that are less critical when using an oil objective. A second explanation could be that there are substantial differences of the optical wave fronts, even though the manufacturers specify equivalent collection angles.

The acquired antibunching curve ${ }^{5}$ (see inset in Fig. 5a) clearly shows that we have a single emitter. The presented $g^{(2)}(\tau)$-function was background corrected as outlined in literature ${ }^{28}$. It further allows to estimate the decay rates of the excited state and metastable triplet state of the NV center. We find the following rates: $T_{1}=9 \mathrm{~ns}, T_{m}=236 \mathrm{~ns}$, which is in good agreement with previous studies. To quantify the achievable count rate, we measure a saturation curve shown Fig. 5a). The low intensity part of the data is well described by

$$
I_{e m}=I_{\mathrm{inf}} \frac{I_{0}}{I_{0}+I_{\text {sat }}},
$$

where $I_{e m}$ is the emitted intensity, $I_{0}$ is the incident intensity, $I_{s a t}$ is the saturation intensity and $I_{\text {inf }}$ is the saturated fluorescence intensity. The above model commonly describes a two-level system, a three-level system with a long lived metastable singlet level, a five-level system accounting for the different spin states of the NV center, as well as a simple four-level system accounting for optically induced charge state switching between $\mathrm{NV}^{0}$ and $\mathrm{NV}^{-29}$. With realistic parameters, all of these models result in qualitatively the same saturation curve. However, at high excitation powers, these models fail to describe the present data. Indeed, in our data we observe that the fluorescence decreases towards high incident powers. Such behaviour has been reported previously ${ }^{18,30}$ and was attributed to the existence of dark states that could be described by a six-level system. Here we propose two simpler models that allow us to describe the saturation behavior at high incident powers. Note that similar models have been exploited to explain the population dynamics of molecules, chromium ${ }^{31}$ and $\mathrm{SiV}^{32}$ centers in diamond. The models are shown schematically in Fig.5 b) and c). We assume that there exists a higher lying excited state that is populated optically and decays spontaneously either back to the same state or to the ground state, indicated by the dashed arrows (within the limits considered below, both cases result in the same saturation behaviour). The higher lying excited statee behaves like a shelving state. Note that it is 
key that the decay of the higher lying state is spontaneous. This is in contrast to the $\mathrm{NV}^{-}$ to $\mathrm{NV}^{0}$ switching mechanism, where both the ionization and the recovery path are believed to be driven by optical pumping ${ }^{29}$. The higher lying state could be coupled either to the excited state or to the metastable singlet state. Thus, the first model employs a three-level system with ground and excited state and an additional higher lying excited state that can be populated optically from the lower excited state and decays spontaneously (the meta stable state is omitted here, to provide the simplest possible model). The second model employs a four-level system with ground, excited and metastable singlet state and a higher lying excited state that can be populated from the singlet state and decays spontaneously back to the singlet state. The models are characterized by the transition rates between the states, which are the excitation and emission rates between the ground and excited state, the excitation and recovery rates to and from the higher lying state and the population and decay rate of metastable state. The rates are denoted $\gamma_{e x}, \gamma_{e m}, \gamma_{s h}, \gamma_{r e}, \gamma_{p}$, and $\gamma_{d}$, respectively. The excitation rates are proportional to the incident intensity and the corresponding efficiencies: $\gamma_{e x}\left(I_{0}\right)=\eta_{e x} I_{0}$ and $\gamma_{s h}\left(I_{0}\right)=\eta_{s h} I_{0}$. The corresponding rate equations result in saturation curves

$$
I_{e m} \propto \frac{\gamma_{r e} \eta_{e x} I_{0}}{\gamma_{e m} \gamma_{r e}+\gamma_{r e} \eta_{e x} I_{0}+\eta_{e x} \eta_{s h} I_{0}^{2}}
$$

and

$$
I_{e m} \propto \frac{\gamma_{d} \gamma_{r e} \eta_{e x} I_{0}}{\left(\gamma_{p}+\gamma_{e m}\right) \gamma_{d} \gamma_{r e}+\left(\gamma_{p}+\gamma_{d}\right) \gamma_{r e} \eta_{e x} I_{0}+\gamma_{p} \eta_{e x} \eta_{s h} I_{0}^{2}}
$$

for the three- and four-level model, respectively. Both models result in the same expression for the saturation curve with three free constants. We fit the experimental data with this common expression. The result is shown by the blue curve in Fig.5.

We conjecture that quite generally, the introduction of a higher lying excited state that is populated by optical pumping but that decays spontaneously will result in the observed saturation behavior.

\section{CONCLUSION}

In this paper, we describe our procedure to precisely locate single emitters both optically inside a diamond substrate and in a focused ion beam machine. FIB milled holes have proven suitable alignment markers, providing $100 \mathrm{~nm}$ lateral and $500 \mathrm{~nm}$ axial accuracy. We have compared different FIB milling strategies. Milling layers of equal thickness yielded the best 

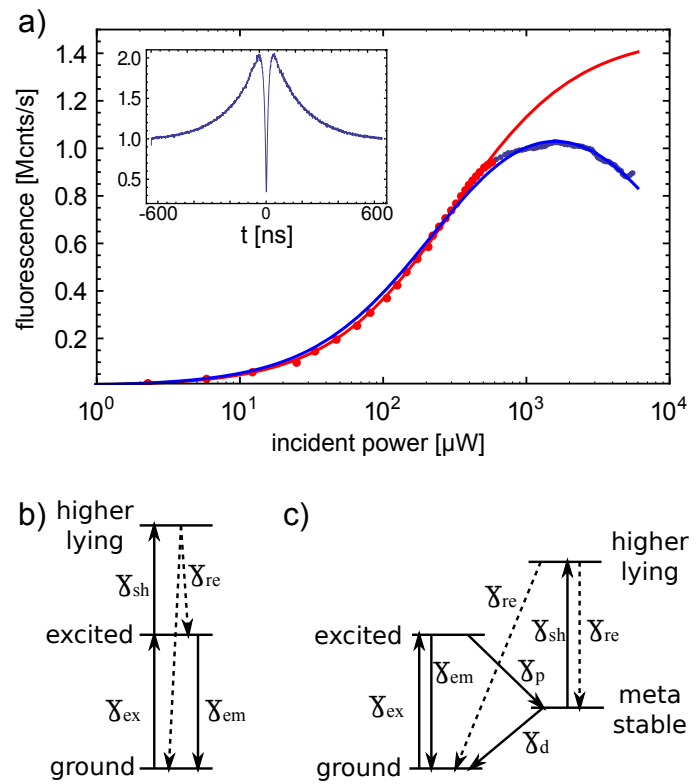

FIG. 5. Saturation behaviour. a) The saturation curve of NV in the center of the SIL (red and blue dots), The red dots show the data up to $550 \mu \mathrm{W}$, the blue dots show the full data set. The red and blue line show fits with a two-level model and a three-level model with higher lying excited state, respectively (see text for details). The inserted plot shows the antibunching at high laser power. b),c) Three- and four-level rate equation models with spontaneously decaying higher lying excited states.

result. This strategy might be applied to other hard materials, to ensure a minimal amount of residuals and optimal feature sharpness. We present record-high count rates of NV at the focus of a SIL. The technique paves the way for high-brightness single photon sources based on solid state emitters and novel spin-control schemes.

\section{ACKNOWLEDGMENTS}

We thank Florian Dolde and Samuel Wagner for help with spin measurements in the SILs and Sen Yang, Thai-Hien Tran and Andrej Denisenko for fruitful discussions. The hospitality of the ITO, Stuttgart is acknowledged. We acknowledge financial support by the Max Planck Society, the ERC project SQUTEC, the DFG SFB/TR21, the EU projects DIAMANT, SIQS, QESSENCE and QINVC, the JST-DFG (FOR1482 and FOR1493), the Volkswagenstiftung and the Landesstiftung Baden-württemberg. 


\section{REFERENCES}

${ }^{1}$ W. E. Moerner and L. Kador, Phys. Rev. Lett. 62, 2535 (1989).

${ }^{2}$ F. Jelezko and J. Wrachtrup, physica status solidi a 203, 3207 (2006).

${ }^{3}$ M. V. G. Dutt, L. Childress, L. Jiang, E. Togan, J. Maze, F. Jelezko, A. S. Zibrov, P. R. Hemmer, and M. D. Lukin, Science 316, 1312 (2007).

${ }^{4}$ R. Brouri, A. Beveratos, J.-P. Poizat, and P. Grangier, Opt. Lett. 25, 1294 (2000).

${ }^{5}$ C. Kurtsiefer, S. Mayer, P. Zarda, and H. Weinfurter, Phys. Rev. Lett. 85, 290 (2000).

${ }^{6}$ G. Balasubramanian, I. Y. Chan, R. Kolesov, M. Al-Hmoud, J. Tisler, C. Shin, C. Kim, A. Wojcik, P. R. Hemmer, A. Krueger, T. Hanke, A. Leitenstorfer, R. Bratschitsch, F. Jelezko, and J. Wrachtrup, Nature 455, 648 (2008).

${ }^{7}$ K. Koyama, M. Yoshita, M. Baba, T. Suemoto, and H. Akiyama, Applied Physics Letters 75,1667 (1999).

${ }^{8}$ K. Lee, X. Chen, E. H., K. P., R. Lettow, A. Renn, V. Sandoghdar, and S. Gotzinger, Nat Photon 5, 166 (2011).

${ }^{9}$ W. Barnes, G. Björk, J. Gérard, P. Jonsson, J. Wasey, P. Worthing, and V. Zwiller, Eur. Phys. J. D 18, 197 (2002).

${ }^{10}$ T. M. Babinec, H. J. M., M. Khan, Y. Zhang, J. R. Maze, P. R. Hemmer, and M. Loncar, Nat Nano 5, 195 (2010).

${ }^{11}$ S. M. Mansfield and G. S. Kino, Applied Physics Letters 57, 2615 (1990).

${ }^{12}$ G. Wrigge, I. Gerhardt, J. Hwang, G. Zumofen, and V. Sandoghdar, Nat Phys 4, 60 (2008).

${ }^{13}$ A. N. Vamivakas, M. Atatuere, J. Dreiser, S. T. Yilmaz, A. Badolato, A. K. Swan, B. B. Goldberg, A. Imamoglu, and M. S. Unlu, Nano Letters 7, 2892 (2007), http://pubs.acs.org/doi/pdf/10.1021/nl0717255.

${ }^{14}$ L. Robledo, L. Childress, H. Bernien, B. Hensen, P. F. A. Alkemade, and R. Hanson, Nature 477, 574 (2011).

${ }^{15}$ G. Waldherr, Y. Wang, S. Zaiser, M. Jamali, T. Schulte-Herbrüggen, H. Abe, T. Ohshima, J. Isoya, J. F. Du, P. Neumann, and J. Wrachtrup, Nature 506, 204 (2014).

${ }^{16}$ R. Kolesov, K. Xia, R. Reuter, M. Jamali, R. Stöhr, T. Inal, P. Siyushev, and J. Wrachtrup, Phys. Rev. Lett. 111, 120502 (2013). 
${ }^{17}$ B. Naydenov, F. Dolde, L. T. Hall, C. Shin, H. Fedder, L. C. L. Hollenberg, F. Jelezko, and J. Wrachtrup, Phys. Rev. B 83, 081201 (2011).

${ }^{18}$ P. Siyushev, F. Kaiser, V. Jacques, I. Gerhardt, S. Bischof, H. Fedder, J. Dodson, M. Markham, D. Twitchen, F. Jelezko, and J. Wrachtrup, Applied Physics Letters 97, 241902 (2010).

${ }^{19}$ L. Marseglia, J. P. Hadden, A. C. Stanley-Clarke, J. P. Harrison, B. Patton, Y.-L. D. Ho, B. Naydenov, F. Jelezko, J. Meijer, P. R. Dolan, J. M. Smith, J. G. Rarity, and J. L. O’Brien, Applied Physics Letters 98, 133107 (2011).

${ }^{20}$ M. Baba, T. Sasaki, M. Yoshita, and H. Akiyama, Journal of Applied Physics 85, 6923 (1999).

${ }^{21}$ N. Bobroff, Review of Scientific Instruments 57, 1152 (1986).

${ }^{22}$ R. E. Thompson, D. R. Larson, and W. W. Webb, Biophysical Journal 82, 2775 (2002).

${ }^{23}$ K. Carlsson, Journal of Microscopy 163, 167 (1991).

${ }^{24}$ S. Hell, G. Reiner, C. Cremer, and E. H. K. Stelzer, Journal of Microscopy 169, 391 (1993).

${ }^{25}$ T. D. Visser, J. L. Oud, and G. J. Brakenhoff, Optik 90, 17 (1992).

${ }^{26}$ K. Ohno, F. Joseph Heremans, L. C. Bassett, B. A. Myers, D. M. Toyli, A. C. Bleszynski Jayich, C. J. Palmstrøm, and D. D. Awschalom, Applied Physics Letters 101, 082413 (2012).

${ }^{27}$ M. Widmann, S.-Y. Lee, T. Rendler, N. T. Son, H. Fedder, S. Paik, N. Zhao, S. Yang, I. Booker, A. Denisenko, M. Jamali, S. A. Momenzadeh, T. Ohshima, A. Gali, E. Janzén, and J. Wrachtrup, arXiv:1407.0180 [cond-mat] (2014), arXiv: 1407.0180.

${ }^{28}$ S. C. Kitson, P. Jonsson, J. G. Rarity, and P. R. Tapster, Phys. Rev. A 58, 620 (1998).

${ }^{29}$ K. Beha, a. Batalov, N. B. Manson, R. Bratschitsch, and a. Leitenstorfer, Physical Review Letters 109, 097404 (2012).

${ }^{30}$ K. Y. Han, D. Wildanger, E. Rittweger, J. Meijer, S. Pezzagna, S. W. Hell, and C. Eggeling, New Journal of Physics 14, 123002 (2012).

${ }^{31}$ I. Aharonovich, S. Castelletto, D. a. Simpson, a. D. Greentree, and S. Prawer, Physical Review A 81, 1 (2010).

${ }^{32}$ E. Neu, M. Agio, and C. Becher, Optics express 20, 19956 (2012), arXiv:1107.0502. 